\title{
Asthma and risk of bronchiectasis exacerbation: we still need more evidence
}

\author{
To the Editor:
}

We have read with great interest the report by MAO et al. [1] investigating the effect of coexisting asthma on the risk of bronchiectasis exacerbation. The investigators reported that the risk of bronchiectasis exacerbation is increased in patients concomitant with asthma compared to those without, with an estimated odds ratio of 2.6 (95\% CI 1.15-5.88). Other variables associated with this relationship included age, duration of symptoms, forced expiratory volume $1 \mathrm{~s}<50 \%$, isolation of Pseudomonas aeruginosa in sputum and lung lesion extension to more than two lobes. Although these are exciting findings, there are some concerns we would like to address.

First, bronchiectasis and asthma share common characteristics including cough, chest tightness, breathless, susceptibility to recurrent exacerbation driven by bacterial or viral infection and development in childhood or adulthood [2-4]. Our previous studies have shown that reversible airway obstruction or hyperresponsiveness can occur in patients with bronchiectasis [5]. Meanwhile, most asthma patients with bronchiectasis had severe persistent asthma in which the reversibility might be limited [6]. All of these factors may result in a diagnostic challenge of asthma in bronchiectasis. Therefore, the method of defining patients with both bronchiectasis and asthma should be documented in detail, which was the foundation of this study [1].

Secondly, the authors claimed that when determining the factors associated with bronchiectasis exacerbation, the variables that presented statistically significant differences $(p<0.05)$ and were of interest were included in the logistic regression model [1]. This suggests the exacerbation in their study was the number of patients experiencing one or more exacerbations but not the frequency of exacerbations (the rate of exacerbation per patients per year) throughout the study period. It is extremely important to understand the difference between the two concepts, which might affect the final results [7]. For example, 60 patients in their study with both bronchiectasis and asthma had at least one exacerbation but only reported 60 exacerbation events, whereas 37 patients with bronchiectasis only had at least one exacerbation but reported 100 exacerbations during the study period (97 patients have at least one exacerbation as presented in the study). Under this condition, the presence of asthma might increase the risk of more patients experiencing one or more exacerbation but not the rates of exacerbation. Therefore, it would be of great interest if MAO et al. [1] could provide some precise answers. 1) How many patients had at least one exacerbation in bronchiectasis alone and concomitant with asthma respectively? 2) Did the relationship between the presence of asthma and increased risk of exacerbation still exist when data were analysed using exacerbation rates by Poisson and negative binomial analyses?

Thirdly, the authors demonstrated that forced expiratory volume in $1 \mathrm{~s}<50 \%$ predicted was independently associated with bronchiectasis exacerbation (OR 4.03, 95\% CI 1.75-9.26; $\mathrm{p}=0.001$ ). The findings per se were not novel [8]. However, the data were collected retrospectively from inpatients, most of which often have exacerbations or haemoptysis in China according to our experience. Therefore, baseline forced expiratory volume in $1 \mathrm{~s}$ could be lower in the study than those collected at steady state, especially in patients concomitant with asthma [9]. In addition, exacerbation per se might increase the airway reversibility in chronic airway disease as previously demonstrated in chronic obstructive pulmonary disease [10], which might result in mislabelling some patients as concomitant with asthma. All of these could be a serious bias for the final conclusion.

Finally, MAO et al. [1] claimed they recorded exacerbations in patients with bronchiectasis using telephone or face-to-face interviews. What I want to know is: how often did the author contact the participants and what was the proportion of the telephone interviews? Because a significant proportion of exacerbations will be unreported using telephone interviews, especially with a long interval, this will lead to underestimation of the incidence and bias the results. These confounding factors, as we mentioned earlier, should be explained before arriving at the final conclusion. 
Yong-hua Gao', Wei-jie Guan ${ }^{2}$, Rong-chang Chen ${ }^{2}$ and Guo-jun Zhang ${ }^{1}$

${ }^{1}$ Dept of Respiratory and Critical Care Medicine, The First Affiliated Hospital of Zhengzhou University, Zhengzhou, China. ${ }^{2}$ State Key Laboratory of Respiratory Disease, National Clinical Research Center for Respiratory Disease, Guangzhou Institute of Respiratory Diseases, The First Affiliated Hospital of Guangzhou Medical University, Guangzhou, China.

Correspondence: Guo-jun Zhang, Dept of Respiratory and Critical Care Medicine, The First Affiliated Hospital of Zhengzhou University, 1 Jianshe East Road, Zhengzhou, Henan 450052, China. E-mail: zhanggj1966@sina.com

Received: April 182016 | Accepted: April 202016

Conflict of interest: None declared.

\section{References}

1 Mao B, Yang J-W, Lu H-W, et al. Asthma and bronchiectasis exacerbation. Eur Respir J 2016; 47: 1680-1686

2 Reddel HK, Bateman ED, Becker A, et al. A summary of the new GINA strategy: a roadmap to asthma control. Eur Respir J 2015; 46: 622-639.

3 Pasteur MC, Bilton D, Hill AT. British Thoracic Society guideline for non-CF bronchiectasis. Thorax 2010; 65: Suppl. 1, i1-58.

4 Gao YH, Guan WJ, Xu G, et al. The role of viral infection in pulmonary exacerbations of bronchiectasis in adults: a prospective study. Chest 2015; 147: 1635-1643.

5 Guan WJ, Gao YH, Xu G, et al. Bronchodilator response in adults with bronchiectasis: correlation with clinical parameters and prognostic implications. J Thorac Dis 2016; 8: 14-23.

6 Boyton RJ, Smith J, Jones M, et al. Human leucocyte antigen class II association in idiopathic bronchiectasis, a disease of chronic lung infection, implicates a role for adaptive immunity. Clin Exp Immunol 2008; 152: 95-101.

7 Gao YH, Guan WJ, Xu G, et al. Macrolide treatment in patients with bronchiectasis: more attention should be paid to the number of exacerbations. Pulm Pharmacol Ther 2014; 27: 213-214.

8 Martinez-Garcia MA, de Gracia J, Vendrell Relat M, et al. Multidimensional approach to non-cystic fibrosis bronchiectasis: the FACED score. Eur Respir J 2014; 43: 1357-1367.

9 Guan WJ, Gao YH, Xu G, et al. Characterization of lung function impairment in adults with bronchiectasis. PloS One 2014; 9: e113373.

10 White AJ, O'Brien C, Hill SL, et al. Exacerbations of COPD diagnosed in primary care: changes in spirometry and relationship to symptoms. COPD 2005; 2: 419-425.

Eur Respir J 2016; 48: 1246-1247 | DOI: 10.1183/13993003.00780-2016 | Copyright (CERS 2016

\section{From the authors:}

According to our study, although more evidence is needed, the existence of asthma is associated with an independent increase in risk of bronchiectasis exacerbation [1]. Bronchiectasis and asthma share some similarities in clinical characteristics and bronchial hyperresponsiveness, which would bring some diagnostic challenges of asthma in bronchiectasis patients. As shown by GuAN et al. [2], reversible airway obstruction or hyperresponsiveness can occur in patients with bronchiectasis. If the extent of reversibility in those patients meets the diagnostic criteria of asthma, they should also be diagnosed with asthma. In our study, the diagnosis and recruitment of subjects was in strict accordance with the relevant guidelines [1, 3-6]. Diagnosis of bronchiectasis was performed using chest high-resolution computed tomography scans in suspected patients with coughing and expectoration, or long durations of haemoptysis. An exacerbation is defined as the patient reporting four or more of the following symptoms: change in sputum production, increased dyspnoea, increased cough, fever $>38^{\circ} \mathrm{C}$, increased wheezing, decreased exercise tolerance, fatigue, malaise, lethargy, reduced pulmonary function, changes in chest sounds, or radiographic changes consistent with a new infectious process $[1,3,4]$. Asthma was diagnosed for patients with symptoms such as episodic breathlessness, wheezing, cough and chest tightness, and whose spirometry showed bronchial reversibility of $12 \%$ and $200 \mathrm{~mL}$ from the pre-bronchodilator value or airway hyperresponsiveness as a $20 \%$ decrease in forced expiratory volume in $1 \mathrm{~s}$ (FEV1) caused by a provocative histamine with a cumulative dose $<2.4 \mathrm{mg}[5,6]$.

After the follow-up, 97 patients had at least one bronchiectasis exacerbation within 1 year after their discharge from the hospital (49 patients with bronchiectasis alone and 48 patients with concomitant asthma). In our study, we used the end-point (exacerbation or not) as a binary variable to make a logistical regression, as other researchers have $[1,7]$. Because of the different durations of follow-up, bronchiectasis exacerbation within 1 year after discharge from the hospital was chosen as the end-point in order to keep consistency. The rate of exacerbation, as usually used in a prospective study, would only conclude the difference of the mean exacerbations of the two groups, and not the hazard ratio of each variable, which was the main purpose of our research. 